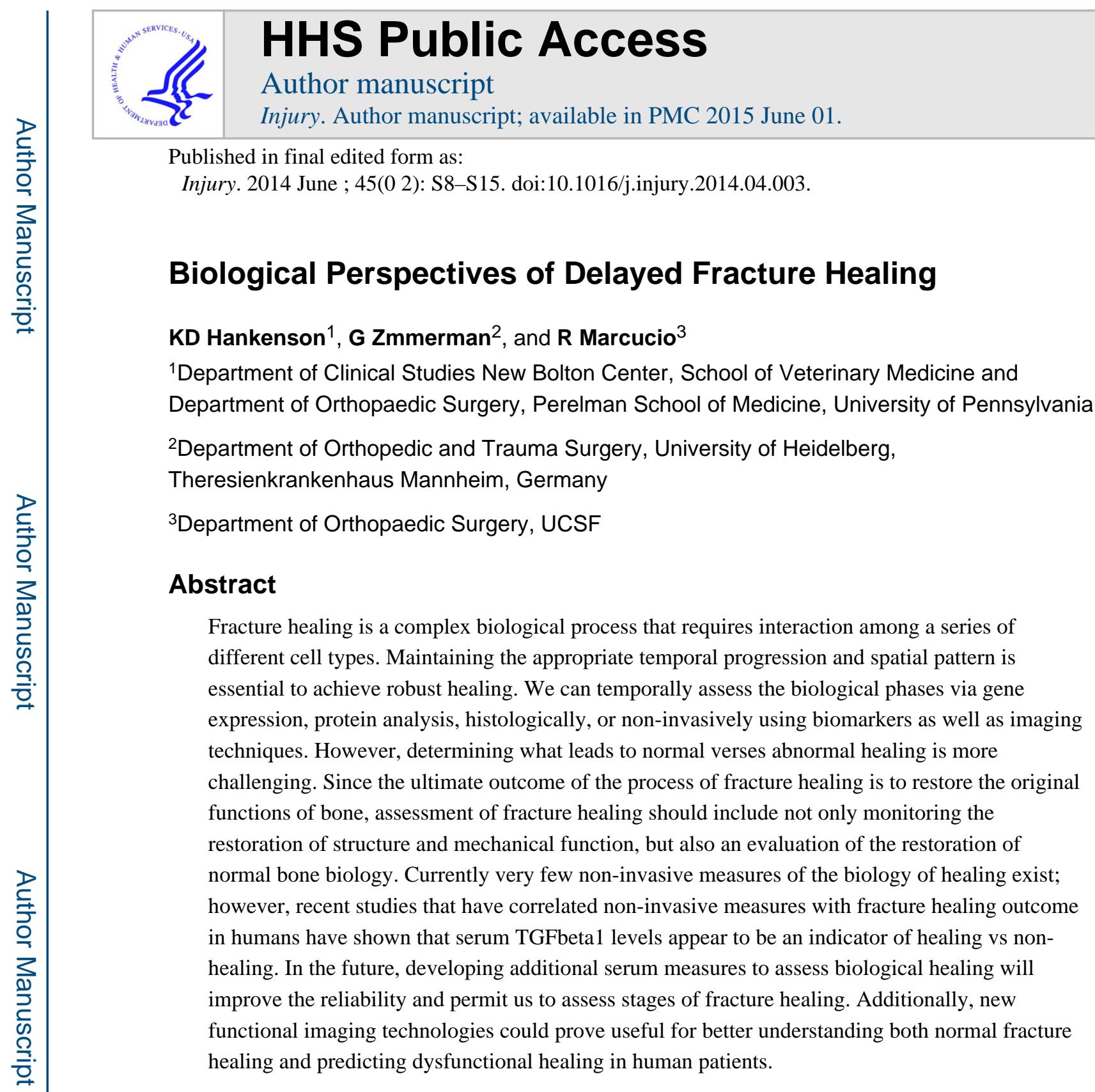

\title{
Introduction
}

The molecular and cellular aspects of fracture healing occur in overlapping stages that have been best described for murine fracture healing [1] (Figure 1). Initially, after injury a hematoma forms and this is followed by inflammatory cells arriving at the fracture site to debride the wound. During the soft callus phase of healing a collar of bone forms at the periosteum adjacent to the fractured bone, and cells in the periosteum proliferate and differentiate into chondrocytes that form cartilage that bridges the fracture gap [2]. During the hard callus phase, the cartilage is replaced by bone through endochondral ossification, possibly by transformation of some of the chondrocytes into osteoblasts [3]. Finally, through remodeling, newly formed bone is restored to its pre-injured state. In mice this entire

Corresponding author: Kurt D. Hankenson, DVM, PhD, 147 Myrin Bldg, 382 West Street Road, Kennett Square, PA 19348, kdhank@vet.upenn.edu. 
process occurs over a period of approximately 28 days, with remodeling continuing for quite some time after that (Fig. 1 and 2).

Recently, this entire sequence of events has been viewed from a different perspective, and the entire healing cascade has been proposed to consist of two distinct phases [4]. First, during the anabolic stage, new bone and cartilage is formed, and during the catabolic phase, the cartilage is replaced by bone which is then further remodeled to restore normal structure. This anabolic-catabolic view cleverly imposes mechanistic actions on the processes during fracture healing and may aid in our understanding of how problem fractures form and in designing therapies to treat these fractures.

In this review article, we consider the various phases of fracture and how alterations in fracture healing may occur from a biological perspective. Next, we consider biological assessments of fracture healing and discuss how we can assess biological factors both invasively and non-invasively to understand the progression of healing in humans with bone fractures, ultimately to both guide treatment and predict fracture outcome.

\section{Role of the inflammatory system in fracture healing}

The influx of inflammatory cells, derived from the bone marrow, is thought to play an important role in the process of fracture healing [5]. However, until recently the importance of inflammatory cells to bone physiology and bone fracture healing have been poorly described. Identification of a population of tissue-resident macrophages that are in close association with osteoblasts lining the bone marrow cavity have been identified and shown to influence normal bone remodeling as well as fracture healing [6]. When macrophage influx to the fracture site is blocked either genetically, or with chemical compounds, fracture healing progresses more slowly [6, 7]. In complementary experiments, stimulating influx of macrophages to the fracture site also stimulates fracture healing. Thus, macrophages appear to play key anabolic roles during fracture healing.

Inflammation may also inhibit bone fracture healing. We have recently shown that sustained inflammation in a mouse model of reduced Notch signaling results in alterations in bone healing characterized by delayed chondrogenesis and reduced callus size [8]. In particular, in humans a number of comorbidities with increased inflammation, including diabetes, smoking, and aging, leads to decreased bone healing potential, but the reasons for the difficulties that are observed are unknown. We tested the ability of juvenile bone marrow to stimulate healing in older mice, and observed that the juvenile bone marrow stimulated bone fracture healing and led to more rapid resolution of inflammation [9]. Our most recent preliminary work demonstrate that blocking macrophage influx into the fracture site in old animals leads to better healing compared to age-matched controls [10]. From these observations, we conclude that the effect of the inflammatory response to bone fracture is age dependent. In young animals macrophages stimulate fracture healing while in older animals macrophages appear to delay fracture healing. We suspect that this is due to changes in the ability of inflammatory cells to polarize from a pro-inflammatory to an antiinflammatory state [11]. 


\section{Role of periosteum in fracture healing}

The periosteum is critical for successful fracture healing because the vast majority of stem and progenitor cells that will form the bone and cartilage regenerate are located in this tissue. In the past, mesenchymal stem cells derived from the systemic circulation were thought to migrate to the site of bone injury and contribute to the formation of bone and cartilage at the fracture site [12]. However, while these studies clearly demonstrated the presence of systemically-derived mesenchymal cells at the fracture site, their contribution to the bone and cartilage was not evident. In contrast, an elegant method for lineage tracing in the callus was used to show that the periosteum and endosteum provided a large majority of cells that formed bone and cartilage at the fracture site [13]. Interestingly, the periosteum had the potential to form both bone and cartilage, but the endosteum was restricted to form only bone [13]. In support of this work, additional lineage tracing in murine fracture that only labels cells with GFP from the alpha-smooth muscle actin promoter at the time of fracture shows the essential contribution of local periosteal cells to the developing callus [14]. Of important note, in both of these studies local cells in the periosteum and endosteum contributed to the bone and cartilage that formed in the fracture callus. That is, the progenitor cells did not migrate from distant sites, rather, they proliferated and then differentiated into chondrocytes and osteoblasts very near their location of origin. While these observations do not rule out the possibility that other cell types may also contribute to the bone and cartilage regenerate, the data underscore the importance of the periosteum for robust fracture healing, because this appears to be the primary tissue of origin of the stem cells that will form both bone and cartilage after fracture.

\section{Role of the vasculature in fracture healing}

Bone is a highly vascularized tissue, and bone formation during development and fracture healing requires a robust angiogenic response [15]. Indeed, surgically induced ischemia in murine models results delayed fracture healing. However, the role(s) that the blood supply plays in fracture healing are not entirely known. Certainly, the blood supply delivers nutrients and some systemically-derived cells to the fracture site. However, recent studies also indicate that the cells of the blood vessels themselves are crucially important in promoting new bone formation. In distraction osteogenesis models of altered hypoxia inducible factor (HIF) signaling, demonstrate that new blood vessels are essential for bone formation [16]. Endothelial cells of blood vessels express members of the Bone Morphogenetic Protein family (BMP) among other signaling molecules to promote fracture regeneration [17]. Incubation of cartilage that is derived from a mouse fracture with human umbilical vein endothelial cells facilitates mineralization of the cartilage in a way that is equal to incubation with BMP2 [3].

Our recent work (R.M and K.H.) has also focused on the role of oxygen during fracture healing. Levels of oxygenation have been associated with increased or decreased chondrogenic and osteogenic potential in vitro. Hypoxia appears to facilitate chondrogenesis $[18,19]$ while higher levels of oxygen appear to support osteogenesis while inhibiting chondrogenesis $[20,21]$. During fracture healing, bone and cartilage form at the fracture site, depending on the amount of fracture site stability [22]. We have determined that non-

Injury. Author manuscript; available in PMC 2015 June 01. 
stabilized fractures have a larger angiogenic response than fractures that are rigidly stabilized [23]. Recently, we directly assessed the effect of oxygen during fracture healing using a number of methods [24, 25]. First, we measured oxygen levels at the fracture site and determined that oxygen levels drop dramatically immediately after fracture, but rapidly return to near normal levels within a few days. Interestingly, during the first few days after injury areas that would eventually support chondrogenesis had lower levels of oxygen than regions where bone would form. To test a causal effect of oxygen levels on bone and cartilage formation, we exposed injured animals to hypoxic, normoxic, or hyperoxic environments. In hypoxia we observed an overall reduction in healing outcomes, but we did not observe a shift in the proportion of cartilage and bone that formed at the fracture site. Similarly, hyperoxia had a negative effect on fracture healing, likely due to the production of superoxide radicals, but we did not observe a shift in the ratio of bone and cartilage at the fracture site. Interestingly, in apparent contrast to work of Wang in distraction osteogenesis [26], we observed that hypoxia led to a decreased angiogenic response compared to normoxic fractures, while hyperoxic fractures had a more robust angiogenic response. While both hypoxia and hyperoxia appear to delay bone fracture healing, hyperoxia actually stimulated healing in animals with an ischemic insult. Ischemic animals housed in a hyperoxic environment had more robust bone and cartilage formation compared to those in normoxic environments. The stimulatory effect in this scenario may be related to the increased stimulatory effect that hyperoxia has on angiogenesis [27]. This effect may lead to increased collateral blood vessel formation and more rapid restoration of blood flow to the lower limb. Hence, therapies that may stimulate angiogenesis may be effective therapies to treat fracture healing, and while this idea has been around for sometime there is little clinical work that has been done to translate this idea to patient care [28].

The experimental data on bone and cartilage formation in vivo are also somewhat in contrast to our computational modeling work in which we demonstrate that alterations in oxygenation impact whether undifferentiated progenitor cells develop into chondrocytes or osteoblasts [25]. Considered together, it is clear that vascularization and oxygenation are undoubtedly essential for bone formation, but ideal levels required for proper healing are still poorly understood.

\section{Role of cell differentiation and endochondral and intramembranous ossification}

The phases of inflammation, angiogenesis, and mesenchymal activation precede the dynamic stage whereby undifferentiated mesenchymal cells undergo both chondrocyte and osteoblast differentiation prior to the process of endochondral and intramembranous ossification. As we have discussed, cells from the periosteum and to some degree the endosteum are the primary contributors to this stage of healing and further, this phase is influence by both the inflammatory phase and vascularization. During chondrogenic differentiation, MSC produce a matrix that is rich in type II collagen and proteoglycans such as aggrecan. As the chondrocytes undergo terminal differentiation they produce type $\mathrm{X}$ collagen and begin to undergo mineralization. Osteoblasts also have a distinct pattern of 
protein production, and produce a spectrum of ECM proteins that are characteristic of differentiated osteoblasts.

This differentiation phase results in a massive expansion of the callus dimension and begins to provide some initial stability. The differentiation of multipotent cells to either the chondrogenic or osteoblastic lineages in the fracture could be regulated by a wide variety of mediators that are known to regulate these processes. In so much as endochondral ossification and intramembranous ossification processes in fracture are akin to these same processes in development, signaling factors that are known to play important roles in development can be used to inform our understanding of bone regeneration. In that regard, Wnts and the TGFbeta super family members TGFbeta and BMPs, are likely important regulators. As well, growth regulators such as Insulin-like growth factor, FGF family members, PTHrP, Hedgehog signaling, and Notch signaling, as well as other growth factors could all impact the process of cell differentiation, maturation, and terminal bone development. However, other non-growth factor proteins, including extracellular matrix proteins and matrix metalloproteinases may also influence the differentiation process. As one example, the genetic disruption of the ECM protein thrombospondin-2 results in undifferentiated cells becoming osteoblasts preferentially over chondrocytes, thereby more bone is formed earlier, and there is earlier provisional callus stability [29].

\section{Role of remodeling}

Following the formation of primary bone, the remodeling phase is characterized by osteoclastic resorption and secondary mature bone formation via intramembranous bone formation. At this stage of bone formation, there is no more chondrogenesis and bone becomes denser and more mature, and there is an eventual restoration of bone shape. The remodeling phase is actually the longest phase of healing, and this is the period through which there is a restoration of both mechanical and biological function. While the extent of mature bone and remodeling changes in shape can be evaluated longitudinally with radiographs and $\mathrm{CT}$, it is also important to understand when biological function is restored.

\section{Assessing the biology of fracture healing}

One dominant theme in the consideration of the phases of fracture healing is the idea that each phase is influenced by preceding phases, thus in any attempt to understand the biology of fracture healing, and assessing the biological stage of healing, it is essential to consider and understand what has happened earlier. Thus, by extension, in assessing fracture healing it is imperative to consider how we can monitor the temporal phases of healing and an eventual restoration of function.

Invasive (destructive) studies to assess fracture healing include evaluating structure, cells, cell and ECM biochemistry, and molecular regulation. Histology - both light microscopic and ultrastructural histological analysis - provides essential information as to when a fracture is healed biologically. Histology can be combined with functional evaluations, antibody based methods or in situ hybridization to discern molecules in a spatial context. While feasible, less work has focused on evaluating the spectrum of proteins produced during fracture healing (proteome), but there has been relatively extensive gene profiling of 
the transcriptome associated with fracture under both normal healing conditions and during altered fracture healing [30]. Even at the single gene level, alterations in gene expression in the heterogenous callus coincide with defined stages of healing (Figure 3). For instance, high-throughput methods have been used to assess altered healing in association with aged fracture healing. These studies show alterations in a transcriptional program in inflammatory mediators (Ahn and Hankenson, unpublished results). Ultimately, the spatial complexity of the healing process, coupled with the overlapping phases of healing make developing a thorough understanding of the biological processes of healing difficult. Ultimately, we will need to develop an extensive molecular, proteomic, and cellular phenotypic map of cell type-specific expression patterns in a temporally defined manner and ultimately normal and abnormal healing must be compared in this context.

Unfortunately, while these invasive measures of cellular, molecular, and proteomic characterization provide essential increases in understanding of fracture biology as well as reveal new potential targets for analysis, in most cases these tools cannot be used in humans, thus non-invasive methods of assessment are required.

\section{Non-invasive approaches to assess the phases of fracture healing}

Non-invasive methods to assess the local inflammatory response in fracture healing and both its magnitude and length are not well-described, but it is conceivable that changes in systemic factors could be assessed. For instance, the profile of cytokines produced locally but expressed systemically could be assessed using serological assays. While some cytokine profiling has been described during experimental fracture healing in rats locally [31] and mice systemically [32], more work would be required to discern systemic patterns of cytokine expression and how those are associated with both normal and abnormal healing.

Despite the essential role of the periosteum in regeneration, methods to measure an appropriate periosteal response or alterations in a periosteal response in the healing process are less clear. Animal models permit us to harvest fractures at various time points and to evaluate those fractures using histological techniques, but obtaining cellular level detail of periosteal responsiveness in humans, in a noninvasive manner, is much more difficult. Thus it would be essential to develop surrogate measures of responsiveness. To date, we have little understanding of cytokines and/or growth factors that may drive surface-specific activation of the periosteum, thus enhanced research in animal models will be required to identify key regulators of this phase of healing.

Being able to assess vascularization and oxygenation to assess fracture healing in human patients could be extremely useful. Potential methodologies for assessing proper vascularization and blood flow include traditional imaging modalities such as angiography, but newer approaches, including blood flow measurement using laser Doppler, nuclear imaging, MRI, Electron paramagnetic spectroscopy (EPR) and even optical imaging could be extremely useful as tools to assess bone healing non-invasively.

Understanding the balance between endochondral and intramembranous bone formation, and the magnitude of each process is critical for assessment of fracture outcome. The pattern of intramembranous and endochondral bone formation is useful for defining the biology of 
healing, and determining when the formation period is completed. Obviously the extent/ magnitude of each of these processes will determine the size of the callus and mechanical stability.

The majority of currently available non-invasive methodologies for assessing healing are focused on evaluating either the differentiation phase (factors regulating differentiation) or the production of extracellular matrix components involved in either endochondral or intramembranous bone formation. From this perspective, the process of intramembranous bone formation can be followed and the process of resorption can be assessed. New bone formation can be assessed similarly to that described for differentiation, based on signaling factors that induce osteoblastogenesis, as well as an increase in osteoblast metabolic activity. On the other hand, osteoclast activity can be assessed by both evaluating factors that regulate osteoclast development such as RANKL and osteoprotegerin, key metabolites of osteoclasts such as tartrate-resistant acid phosphatase (TRAP) and cathepsin K, and breakdown products of bone ECM, such as type I collagen. Again, the ability to assay for proteins in human clinical cases is primarily limited to those factors that can be assessed using serum or urine biomarkers.

\section{Utilizing systemic biological markers to assess delayed and non-union healing}

Few studies have evaluated either imaging methods or laboratory-based methods to predict normal and prolonged healing, specifically of long-bone fractures. Systemically measured biological markers may facilitate the earliest diagnosis of delayed union. As previously described, biological markers can represent the release of components of the extracellular matrix that are either produced or degraded during the remodeling phase, or can include factors produced locally or systemically, such as growth factors, that guide the normal healing process.

Markers of bone formation are predominantly derived from osteoblasts. Current serum markers of bone formation activity include bone-specific alkaline phosphatase (ALP), procollagen type-I N-terminal propeptide (PINP), procollagen type-I C-terminal propeptide (PICP), and osteocalcin (OC). Also markers of bone resorption are available, including collagen breakdown products and enzymes such as tartrate resistant acid phosphatase and cathepsin K produced by osteoclasts. A number of studies over the past 15 years have been completed that have prospectively evaluated serum markers of bone formation following fracture. These studies have been done in both humans and animals. For instance, Ingle et al show alterations in AP, OC, and PINP with fracture. What is interesting to note is the wide individual variation, and also the length of time that these changes are manifested [33]. While a variety of studies show alterations (elevations) in a variety of markers, the usefulness of the markers is limited, without an understanding of the concurrent biological processes and the phases of healing. As well, markers that have been studied in bone regeneration reflect only the bone formation phase, and as previously discussed, in many cases we may need to understand earlier events. 
There are also non-matrix components that have been studied in serum following fracture. As an example, sclerostin, a Wnt and BMP signaling inhibitor, levels are elevated in human fracture healing [34], and there are elevations in the levels of macrophage colonystimulating factor in human fracture healing [35]

While it is clear that serum markers can be used to demonstrate healing and perhaps to follow temporal changes in healing, the question of their usefulness in assessing poor healing is a more complicated question. To date it is still difficult to predict accurately normal consolidation of bone [36]. In clinical practice plain X-rays and CT are used. The usefulness of the results is limited and often does not correlate with functional healing. Importantly, a "diagnostic gap" of 6-8 weeks exists, during which time the patient and the surgeon are not aware of healing problems. To close this gap and to have a more guided approach in managing fracture many attempts have been made to monitor fracture healing with serological investigations (Table 1). Some of these parameters provide information but only a few can distinguish between healing and no healing.

In a canine study of fracture healing, Komnenou et al show alterations in alkaline phosphatase, an enzyme involved in osteoblastogenesis and bone formation [37]. Both VEGF and TGFbeta-1 show dysregulation in association with altered healing [38, 39], but perhaps the most compelling serum-based component that shows defined alterations in association with poor healing is TGFbeta1 [40]. The scientific background of this test was demonstrated in a prospective controlled study with 103 diaphyseal case-matched fractures of long bone. These patients were controlled over a period of 6 months following a standardized time schedule. The levels of over 80 cytokines, which are known for their influence on bone healing, were determined 1,2, 4, 6, and 8 weeks and 3, 6, and 12 months after the injury. During a study period of 1 year, 15 patients in the initial study with an atrophic type of delayed union were studied and matched to 15 patients with normal fracture healing (Figure 4). The diagnosis of delayed union was assumed in if there was failed consolidation radiogrphaically 4 months after trauma. An increase of TGF- $\$ 1$ serum levels up to 2 weeks after fracture were found in both groups with a return to the reference value within 6 weeks after trauma. However, the decline of serum TGF-beta1 levels occurred earlier in patients with delayed fracture healing. At 4 weeks after trauma, serum levels of TGF-beta1 were significantly lower in patients in the delayed union group. These findings support a critical role of TGF-beta1 in fracture healing and suggest that premature drops in TGF-beta1 levels are indicative of problems with healing. Indeed, eents during the consolidation phase seem to be dependent on sufficient TGF-beta1. These results have now been confirmed with over 30 patients in each group.

There is an important potential clinical impact of these results. In Figure 5 the accuracy of this test is illustrated. With a safety of more than $95 \%$ the likelihood of developing a nonunion over the healing time could be predicted at a time point of 4 weeks after trauma by measuring serum TGF-beta1. Clinically this means that the diagnosis of a non-union can be reliably done after 4 weeks. Therefore, patients could be managed in a very early state with supporting bone healing methods. This could have a significant economic impact as 16 weeks of unemployment because of a non-union costs society at least $\$ 80,000$ [41]. 
Ultimately, a greater number of serum based markers that evaluate the various phases of fracture healing should also be developed. This would increase both the sensitivity and reliability of using biomarkers for determination of healing potential. Approaches to determine new markers to pursue will likely involve high-throughput technologies that study expressed genes (locally) or protein products (locally or systemically). Possible approaches include high-throughput proteomic analysis of serum post-fracture, and then correlating changes with phases of healing. On the other hand, utilizing local transcriptomic and proteomic approaches to identify putative candidates that may be produced locally will require animal models of induced fracture to be able to correlate parameters of healing with other serum markers.

Beyond serum markers, most of the assays that could serve as non-invasive biological predictors of fracture healing are still in a developmental stage. Vascularization is routinely assessed using MRI in combination with gadolinium clinically and this tool could be applied to assess the vascularization of fractures as predictive of healing outcome. This approach has been used to predict healing outcome of scaphoid bone fractures as well as perfusion of the femoral head following femoral neck fracture [42-44]; however, it has not been reportedly used to predict fracture outcome of long-bone fractures.

A new frontier in assessing biological basis for healing is functional imaging. Optical imaging using near-infrared light has been FDA-approved and there are clinical instruments available. Flurophores can be coupled to reagents that can be incorporated into forming bone or that can identify blood vessels. While there are no reports of optical imaging being used for human fracture, Preliminary work has been completed on mouse models showing enhanced optical imaging signal during regeneration [45].

Another possible imaging approach to assess biological approaches to fracture healing is radionuclide imagining with PET with fluorine-18 sodium fluoride or SPECT with technetium-99m methylene diphosphonate. However, radionuclide signals need to be correlated with phases of healing.

Finally, MRI-based imaging approaches that have been used for cartilage could be used to evaluate chondrogenesis and endochondral ossification. T2 mapping, T1rho, sodium MRI, and delayed gadolinium-enhanced MRI of cartilage (dGEMRIC) take advantage of cartilage biochemistry to image cartilage effectively and could be used to evaluate the cartilaginous callus in secondary healing [46].

Despite the promise of these functional imaging techniques as useful tools for healing potential prediction, a great deal of additional work must be completed before the techniques could be used as biological predictors of fracture outcome.

\section{Conclusions}

Developing methods to better assess the biological-basis for fracture healing is essential to improve patient care. The key to this development has two components. First as we have discussed throughout this review, we must better understand the temporal aspects of biological healing and then be able to develop useful assessment strategies in clinical 
patients. From this we can then develop outcome parameters that are predictive of biologically based healing. To be successful, a number of questions must be addressed:

- Non-invasively (non-destructively), can we detect when one phase of fracture healing has ended and another has begun?

- What is the biochemical and molecular profile of fractures that heal relative to those that don't heal and could these profiles reveal 'targets' to analyze to predict outcome?

- How do we most effectively validate the utilization of biomarkers to monitor healing and the possibility of poor healing?

- $\quad$ Can we develop new, more specific, biomarkers for fracture healing?

- $\quad$ Could a spectrum of biomarkers be utilized to predict fracture outcome, as well as, to understand the varying phases of healing?

- Will novel functional imaging modalities be useful to assess biological healing?

Addressing these questions will undoutedly require more investigations in animal models of altered fracture healing that focus on development of non-invasive methodoliges that can then be extend to the human clinical patient. These results can then be cross-compared with perspective studies of human fracture samples - both tissue harvests as well as serum harvests - that then can be correlated with fracture outcome.

\section{Acknowledgments}

RM is supported by 1R01AG046282-01.

\section{References}

1. Einhorn TA. The cell and molecular biology of fracture healing. Clin Orthop. 1998; (355 Suppl):S7-21. [PubMed: 9917622]

2. Colnot C, Zhang X, Knothe Tate ML. Current insights on the regenerative potential of the periosteum: molecular, cellular, and endogenous engineering approaches. J Orthop Res. 2012; 30(12):1869-78. [PubMed: 22778049]

3. Bahney CS, et al. Stem Cell Derived Endochondral Cartilage Stimulates Bone Healing by Tissue Transformation. J Bone Miner Res. 2013

4. Little DG, Ramachandran M, Schindeler A. The anabolic and catabolic responses in bone repair. J Bone Joint Surg Br. 2007; 89(4):425-33. [PubMed: 17463107]

5. Colnot C, Huang S, Helms J. Analyzing the cellular contribution of bone marrow to fracture healing using bone marrow transplantation in mice. Biochem Biophys Res Commun. 2006; 350(3):557-61. [PubMed: 17022937]

6. Alexander KA, et al. Osteal macrophages promote in vivo intramembranous bone healing in a mouse tibial injury model. J Bone Miner Res. 2011; 26(7):1517-32. [PubMed: 21305607]

7. Xing Z, et al. Multiple roles for CCR2 during fracture healing. Dis Model Mech. 2010; 3(7-8):4518. [PubMed: 20354109]

8. Dishowitz MI, et al. Inhibition of canonical Notch signaling results in sustained callus inflammation and alters multiple phases of fracture healing. PLoS ONE. 2013 in press.

9. Xing Z, et al. Rejuvenation of the inflammatory system stimulates fracture repair in aged mice. J Orthop Res. 2010; 28(8):1000-6. [PubMed: 20108320] 
10. Slade Shantz JA, et al. Modulation of Macrophage Activity During Fracture Repair has Differential Effects in Young Adult and Elderly Mice. J Orthop Trauma. 2013

11. Sebastian C, et al. MacrophAging: a cellular and molecular review. Immunobiology. 2005; $210(2-$ 4):121-6. [PubMed: 16164018]

12. Kumagai K, et al. Circulating cells with osteogenic potential are physiologically mobilized into the fracture healing site in the parabiotic mice model. J Orthop Res. 2008; 26(2):165-75. [PubMed: 17729300]

13. Colnot C. Skeletal Cell Fate Decisions Within Periosteum and Bone Marrow During Bone Regeneration. J Bone Miner Res. 2008

14. Grcevic D, et al. In vivo fate mapping identifies mesenchymal progenitor cells. Stem Cells. 2012; 30(2):187-96. [PubMed: 22083974]

15. Lu C, et al. Ischemia leads to delayed union during fracture healing: a mouse model. J Orthop Res. 2007; 25(1):51-61. [PubMed: 17019699]

16. Riddle R, et al. Role of hypoxia-inducible factor-1a in angiogenic-osteogenic coupling. Journal of Molecular Medicine. 2009; 87(6):583-590. [PubMed: 19415227]

17. Jacobsen KA, et al. Bone Formation During Distraction Osteogenesis Is Dependent on Both VEGFR1 and VEGFR2 Signaling. Journal of Bone and Mineral Research. 2008; 23(5):596-609. [PubMed: 18433297]

18. Merceron C, et al. Differential effects of hypoxia on osteochondrogenic potential of human adipose-derived stem cells. American Journal of Physiology - Cell Physiology. 298(2):C355C364. [PubMed: 19940068]

19. Amarilio R, et al. HIF1 \{alpha\} regulation of Sox9 is necessary to maintain differentiation of hypoxic prechondrogenic cells during early skeletogenesis. DEVELOPMENT. 2007; 134(21): 3917-3928. [PubMed: 17913788]

20. Wagegg M, et al. Hypoxia Promotes Osteogenesis but Suppresses Adipogenesis of Human Mesenchymal Stromal Cells in a Hypoxia-Inducible Factor-1 Dependent Manner. PLoS ONE. 7(9):e46483. [PubMed: 23029528]

21. D'Ippolito G, et al. Low oxygen tension inhibits osteogenic differentiation and enhances stemness of human MIAMI cells. Bone. 2006; 39(3):513-522. [PubMed: 16616713]

22. Thompson Z, et al. A model for intramembranous ossification during fracture healing. J Orthop Res. 2002; 20(5):1091-8. [PubMed: 12382977]

23. Lu C, et al. Mechanical stability affects angiogenesis during early fracture healing. J Orthop Trauma. 2011; 25(8):494-9. [PubMed: 21738063]

24. Lu C, et al. The role of oxygen during fracture healing. Bone. 2013; 52(1):220-9. [PubMed: 23063782]

25 . Burke D, et al. The role of oxygen as a regulator of stem cell fate during fracture repair in TSP2null mice. J Orthop Res. 2013

26. Wan $\mathrm{C}$, et al. Activation of the hypoxia-inducible factor-1alpha pathway accelerates bone regeneration. Proc Natl Acad Sci U S A. 2008; 105(2):686-91. [PubMed: 18184809]

27. Sheikh AY, et al. Hyperoxia improves microvascular perfusion in a murine wound model. Wound Repair Regen. 2005; 13(3):303-8. [PubMed: 15953050]

28. Street J, et al. Vascular endothelial growth factor stimulates bone repair by promoting angiogenesis and bone turnover. Proc Natl Acad Sci U S A. 2002; 99(15):9656-61. [PubMed: 12118119]

29. Taylor DK, et al. Thrombospondin-2 Influences the Proportion of Cartilage and Bone During Fracture Healing. J Bone Miner Res. 2009; 24(6):1043-1054. [PubMed: 19123916]

30. Wang K, et al. Analysis of fracture healing by large-scale transcriptional profile identified temporal relationships between metalloproteinase and ADAMTS mRNA expression. Matrix Biol. 2006; 25(5):271-81. [PubMed: 16584876]

31. Einhorn TA, et al. The expression of cytokine activity by fracture callus. Journal of Bone \& Mineral Research. 1995; 10(8):1272-81. [PubMed: 8585432]

32. Kobbe $\mathrm{P}$, et al. Patterns of cytokine release and evolution of remote organ dysfunction after bilateral femur fracture. Shock. 2008; 30(1):43-7. [PubMed: 18562923] 
33. Ingle BM, et al. Changes in Bone Mass and Bone Turnover Following Distal Forearm Fracture. Osteoporosis International. 1999; 10(5):399-407. [PubMed: 10591838]

34. Sarahrudi K, et al. Strongly enhanced levels of sclerostin during human fracture healing. JOURNAL OF ORTHOPAEDIC RESEARCH. 30(10):1549-1555. [PubMed: 22508529]

35. Sarahrudi K, et al. Elevated levels of macrophage colony-stimulating factor in human fracture healing. JOURNAL OF ORTHOPAEDIC RESEARCH. 28(5):671-676. [PubMed: 19950360]

36. Zimmermann G, Muller U, Wentzensen A. The value of laboratory and imaging studies in the evaluation of long-bone non-unions. Injury. 2007; 38(Suppl 2):S33-7. [PubMed: 17920416]

37. Komnenou A, et al. Correlation of serum alkaline phosphatase activity with the healing process of long bone fractures in dogs. Veterinary Clinical Pathology. 2005; 34(1):35-38. [PubMed: 15732015]

38. Sarahrudi K, et al. Elevated transforming growth factor-beta 1 (TGF-Î²1) levels in human fracture healing. Injury. 42(8):833-837. [PubMed: 21529804]

39. Sarahrudi K, et al. Elevated transforming growth factor-beta 1 (TGF-beta1) levels in human fracture healing. Injury. 2011; 42(8):833-7. [PubMed: 21529804]

40. Zimmermann G, et al. TGF-[beta]1 as a marker of delayed fracture healing. Bone. 2005; 36(5): 779-785. [PubMed: 15811636]

41. Sprague S, Bhandari M. An economic evaluation of early versus delayed operative treatment in patients with closed tibial shaft fractures. Arch Orthop Trauma Surg. 2002; 122(6):315-23. [PubMed: 12136294]

42. Kamano M, et al. Contrast enhanced magnetic resonance imaging for femoral neck fracture. Clin Orthop Relat Res. 1998; (350):179-86. [PubMed: 9602818]

43. Dawson JS, Martel AL, Davis TR. Scaphoid blood flow and acute fracture healing. A dynamic MRI study with enhancement with gadolinium. J Bone Joint Surg Br. 2001; 83(6):809-14. [PubMed: 11521919]

44. Megerle K, et al. Gadolinium-enhanced preoperative MRI scans as a prognostic parameter in scaphoid nonunion. J Hand Surg Eur Vol. 2011; 36(1):23-8. [PubMed: 20621936]

45. Zilberman Y, et al. Fluorescence molecular tomography enables in vivo visualization and quantification of nonunion fracture repair induced by genetically engineered mesenchymal stem cells. J Orthop Res. 2008; 26(4):522-30. [PubMed: 17985393]

46. Fleming BC, et al. Delayed Gadolinium-Enhanced MR Imaging of Cartilage (dGEMRIC) following ACL injury. Osteoarthritis Cartilage. 2010; 18(5):662-7. [PubMed: 20188685] 


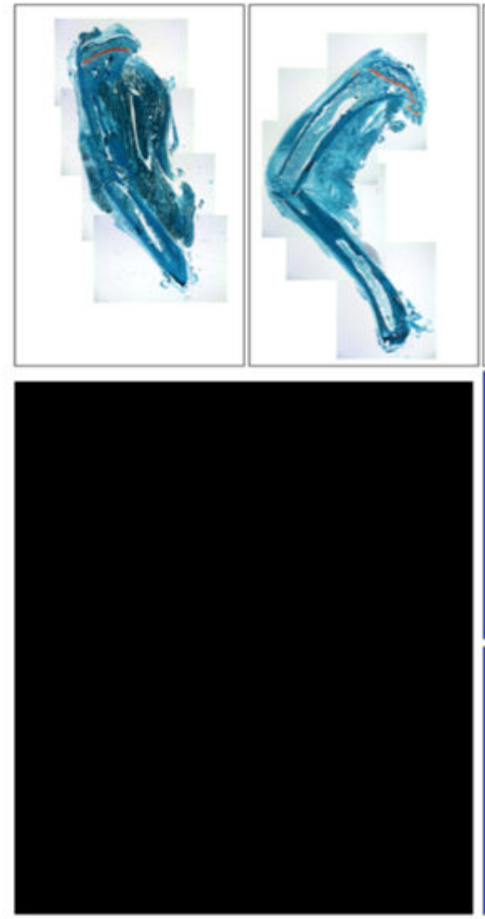

2 DPF
$5 \mathrm{DPF}$

\section{Angiogenesis Mesenchyme}
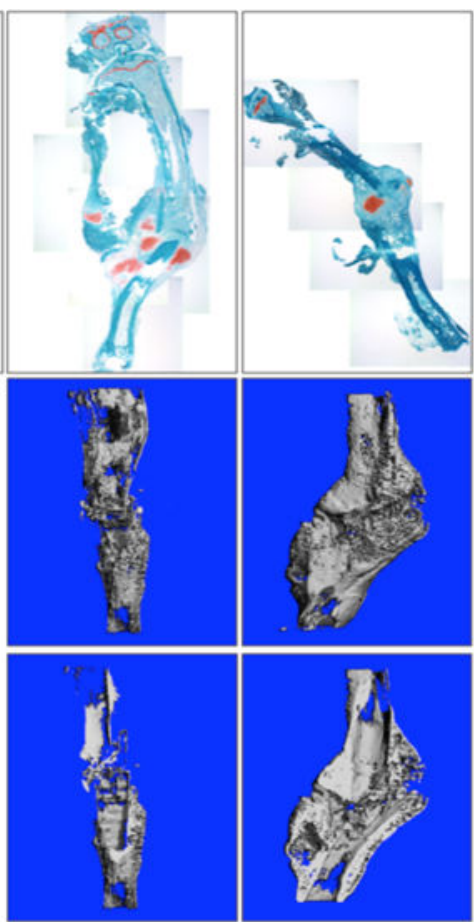

$10 \mathrm{DPF}$

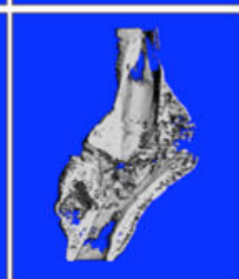

15 DPF
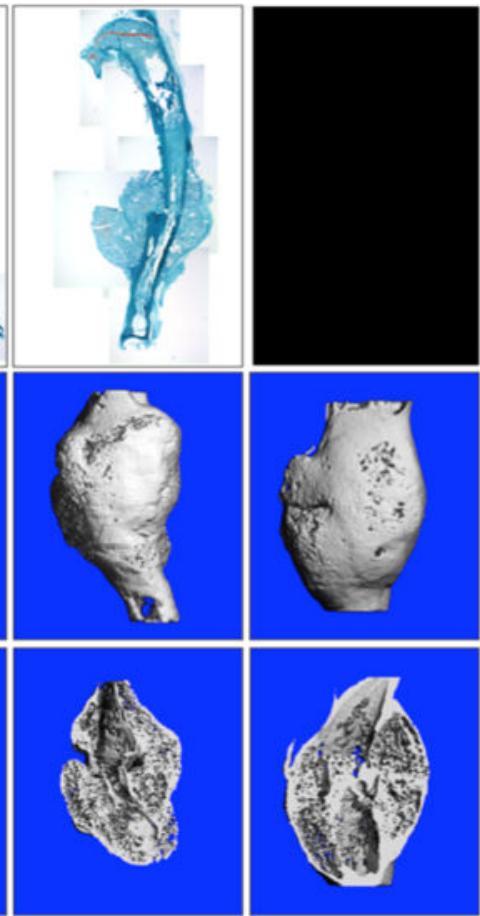

20 DPF

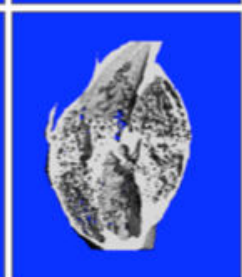

$30 \mathrm{DPF}$

\section{Hematoma}

Inflammation

\section{Mesenchyme Differentiation}

\section{Endochondral \& Intramembranous bone formation}

Figure 1. Tibial shaft fracture in a mouse at days $2,5,10,15,20$, and 30 post-fracture (DPF) Top row are histology sections stained with Fast Green FCF and Safranin-O, and represent a series of $2 \mathrm{X}$ images that have been stitched together to show the entire tibia. Rows 2 and 3 are micro-CT images of the callus; the middle row is a three-dimensional reconstruction of the callus and the bottom row is a coronal section of the specimen. The 2 and 5 DPF time points represent the early inflammatory and marrow response stage. 10 DPF represents the early intermediate phase dominated by chondrogenesis. 15 DPF demonstrates the transition to the late intermediate stage marked by primary bone formation observed at $20 \mathrm{DPF}$. Finally, secondary bone formation and remodeling can be observed at 30 DPF. 


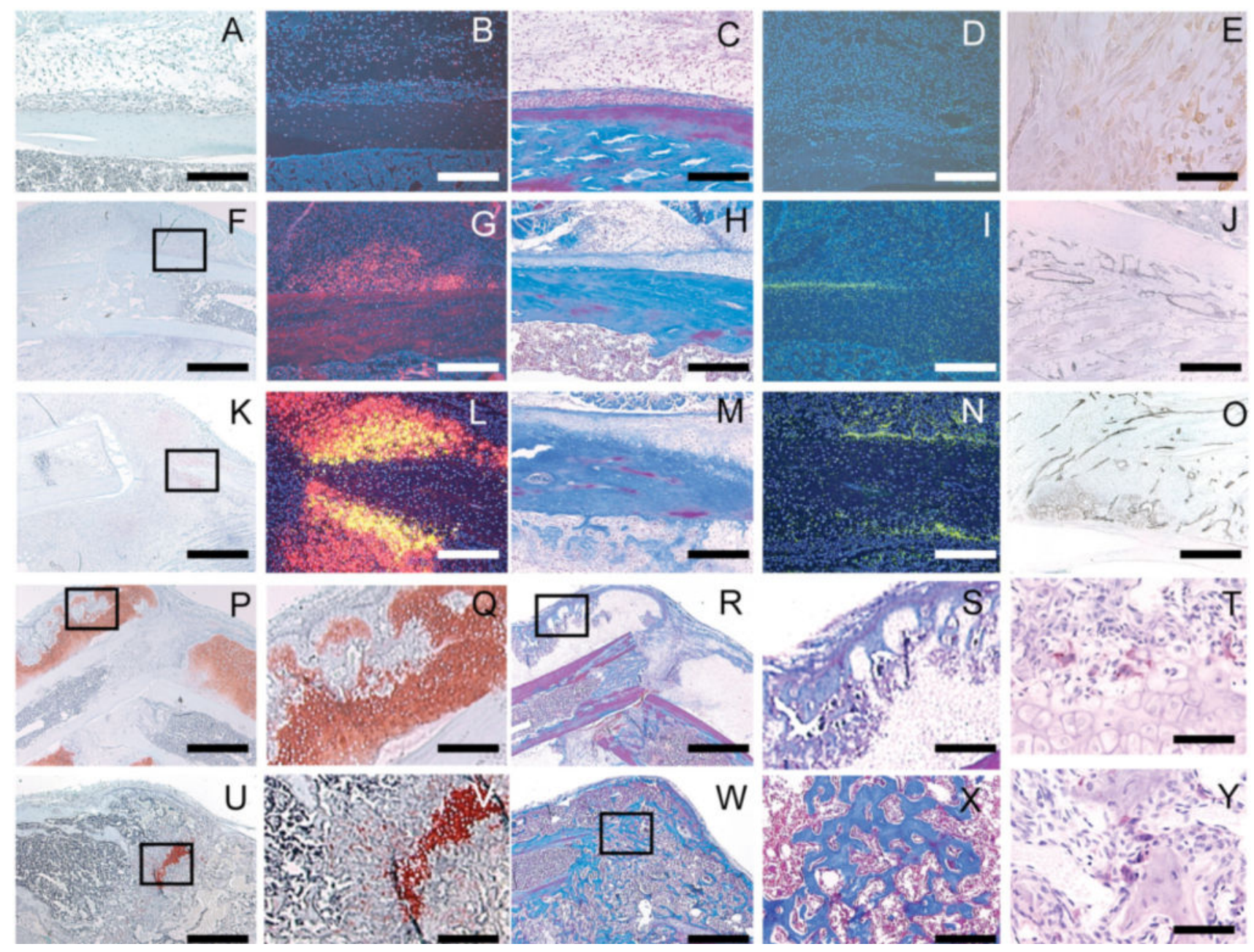

Figure 2. The process of fracture healing in non-stabilized fractures of the mouse tibia Safranin-O/fast green (A,F,K,P,Q,U,V) and trichrome (C,H,M,R,S,W,X) were used to staining cartilage red and bone blue respectively. Transcripts of Col2 (B,G,L), Col10 (L), and osteocalcin $(\mathrm{D}, \mathrm{I}, \mathrm{N})$ were detected by in situ hybridization, pseudocolored red, yellow, and green and used to visualize chondrocytes, hypertrophic chondrocytes, and osteoblasts respectively. Macrophages were detected via immunohistochemistry using antibody F4/80 (E), and endothelial cells were stained blach using immunohistochemistry with an antibody against PECAM (J,O, also known as CD31). Osteoclasts were stained red using tartrateresistant acid phosphatase (T,Y). (A) At day 3 post fracture no cartilage or (B) Col2/Col10 transcripts were detected. (C) No bone or (D) osteocalcin was present. (E) At this time, macrophages have infiltrated the fracture site. (F) At 5 days, immature cartilage (G) expressing Col2 but not Col10 was observed in the periosteum. $(\mathrm{H}) \mathrm{A}$ small amount of new bone (I) and osteocalcin expression was also apparent in the periosteal reaction, (J) which is highly vascularized. (K,1) At 7 days, cartilage is beginning to mature, and Col10 transcripts are evident. (M,N) More new bone and osteocalcin expression are also evident. (O) Vascular invasion was observed around hypertrophic chondrocytes. (P,Q) At day 14 a large amount of cartilage and (R,S) bone were formed. (T) Multiple osteoclasts were observed at the front of endochondral ossification. (U,V) By day 21 after injury, cartilage has been replaced by 
bone. (W,X) Fractures have healed by bony bridging. (Y) Osteoclasts on the surface of trabecular bone continue to remodel the newly formed bone. Scale bar: A-D, G-I, L-O, $\mathrm{Q}, \mathrm{S}, \mathrm{V}$, and $\mathrm{X}=250 \mathrm{~mm} ; \mathrm{E}, \mathrm{T}$, and $\mathrm{Y}=60 \mathrm{~mm}, \mathrm{~F}, \mathrm{~K}, \mathrm{P}, \mathrm{U}, \mathrm{R}=1 \mathrm{~mm}$. 


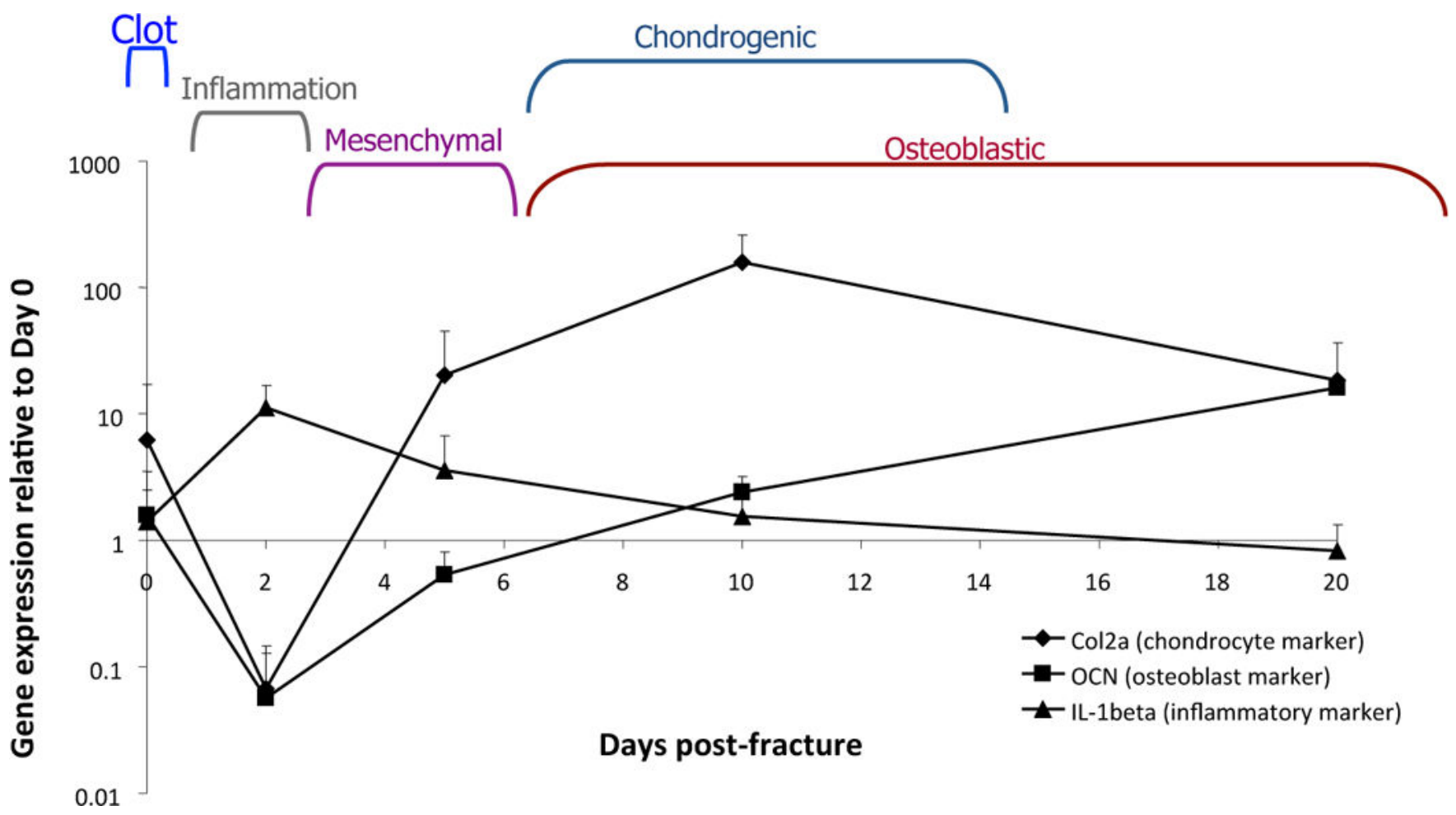

Figure 3. Changes in whole-callus gene expression reflect the rapidly changing temporal biology of fracture healing

RNA was harvested from murine calluses following fracture and assessed for markers of inflammation (IL1-beta), chondrogenesis (type 2 collagen), and bone formation (osteocalcin). 


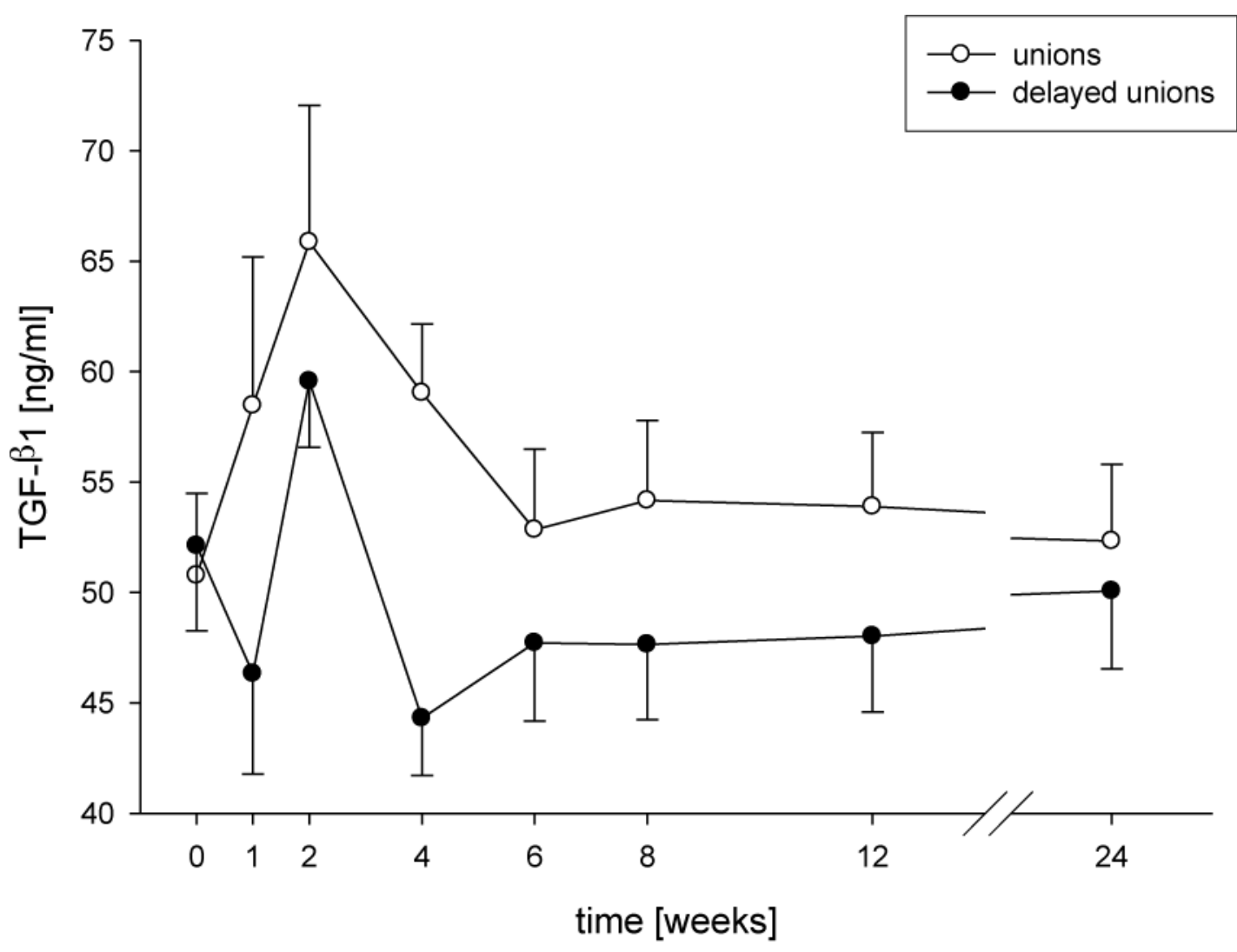

Figure 4. TGF-b1 serum levels presented as absolute concentrations

Time course of concentrations of TGF-b1 (mean + SEM) from patients after surgery for long-bone shaft fractures with normal (unions) or delayed consolidation (delayed unions). Significant changes compared to the reference value (48-week unions; 72-week delayed unions) are indicated by asterisks ( $* \mathrm{P}<0.05$, $* * \mathrm{P}<0.01)$. \# Indicates significant differences a certain point between the union and delayed union group (\#P $<0.05$, \#\# $<0.01)$. Clamps illustrate significant time related changes within both groups. 


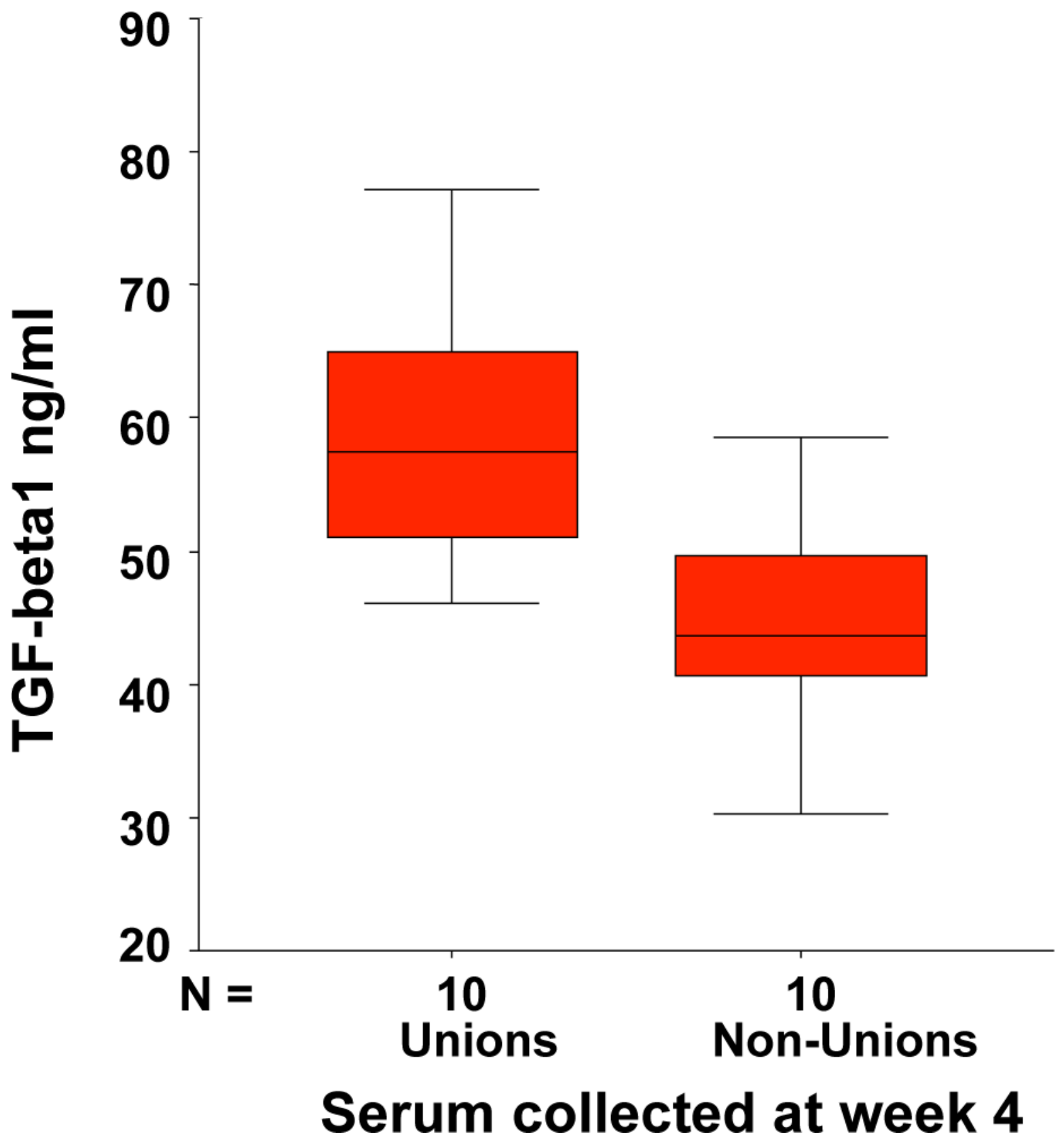

Figure 5. Decreases in serum TGF-b1 levels reflect a failure to heal

Based on a number of 15 patients in each group the distribution of the values in the group of non unions and unions could be seen. At a level of under $45 \mathrm{ng} / \mathrm{cc}$ TGFß-1 only one patient of 15 would have healed in the next months and only one patient over $45 \mathrm{ng} / \mathrm{cc}$ of 15 developed a non union. 


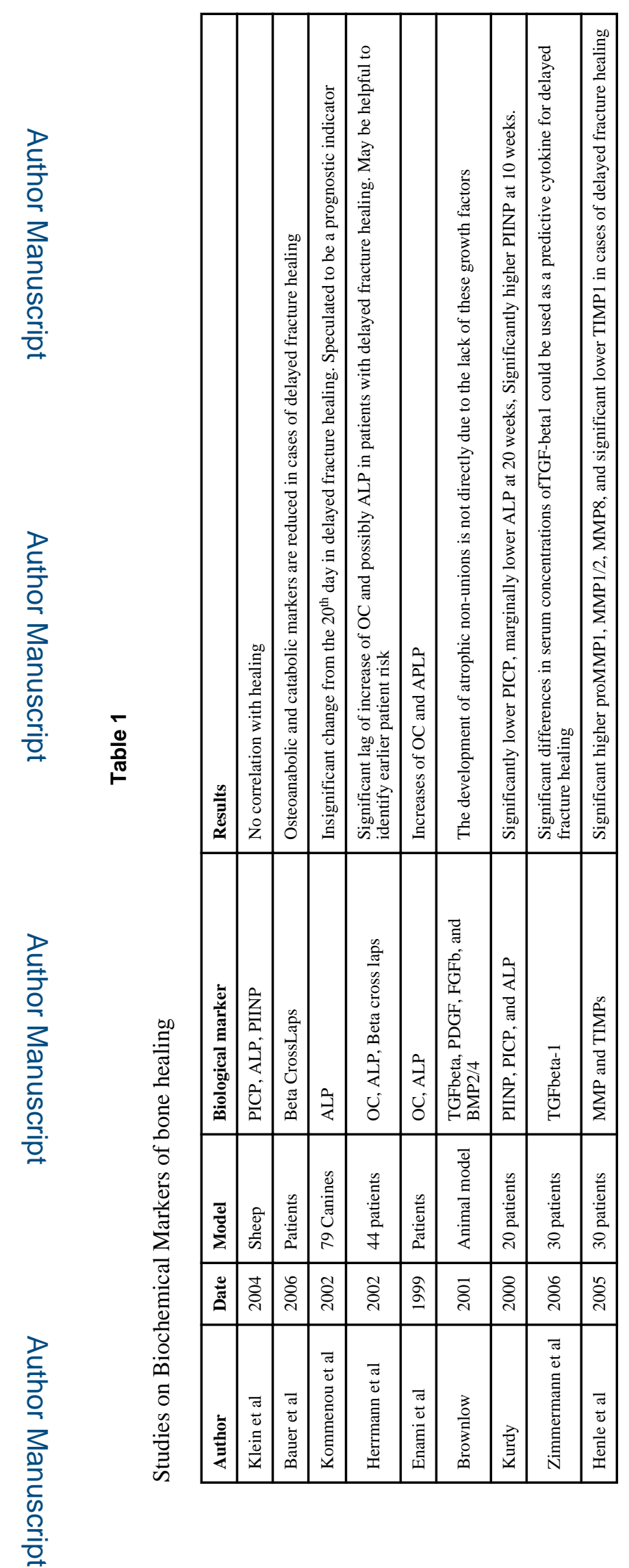

Injury. Author manuscript; available in PMC 2015 June 01. 